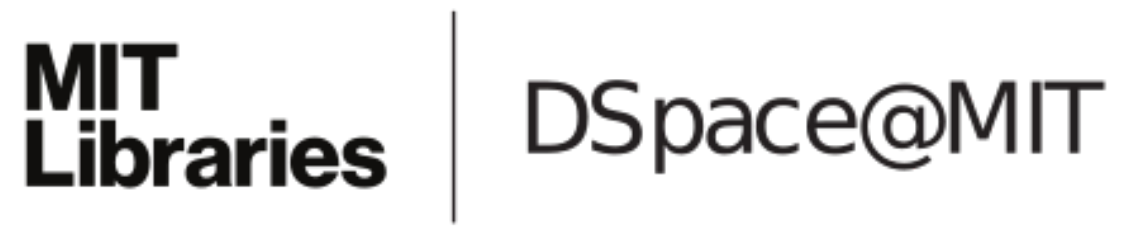

\author{
MIT Open Access Articles
}

Droplet fragmentation using a mesh

The MIT Faculty has made this article openly available. Please share how this access benefits you. Your story matters.

Citation: Soto, Dan et al. “Droplet Fragmentation Using a Mesh.” Physical Review Fluids 3, 8 (August 2018): 083602 (C) 2018 American Physical Society

As Published: http://dx.doi.org/10.1103/PhysRevFluids.3.083602

Publisher: American Physical Society

Persistent URL: http://hdl.handle.net/1721.1/120136

Version: Final published version: final published article, as it appeared in a journal, conference proceedings, or other formally published context

Terms of Use: Article is made available in accordance with the publisher's policy and may be subject to US copyright law. Please refer to the publisher's site for terms of use. 


\title{
Droplet fragmentation using a mesh
}

\author{
Dan Soto, ${ }^{1}$ Henri-Louis Girard, ${ }^{1}$ Antoine Le Helloco, ${ }^{1}$ Thomas Binder, ${ }^{1}$ \\ David Quéré, ${ }^{2, *}$ and Kripa K. Varanasi ${ }^{1, \dagger}$ \\ ${ }^{1}$ Department of Mechanical Engineering, MIT, Cambridge, MA 02139, USA \\ ${ }^{2}$ Physique et Mécanique des Milieux Hétérogènes, UMR 7636 du CNRS, \\ École Supérieure de Physique et de Chimie Industrielles, 75005 Paris, France
}

(Received 15 March 2018; published 28 August 2018)

\begin{abstract}
Atomization and spray generation naturally occur around us in a wide variety of situations ranging from drop impacts to bubble bursting. However, controlling this process is key in many applications such as internal combustion engines, gas turbines, and agricultural spraying. Here we show how a drop can be fragmented into thousands of smaller droplets by impacting it onto a mesh. We demonstrate the unexpected possibility to transfer liquid outside the projected impact area of the drop and the existence of a well-defined cone envelope for the resulting spray. Self-similarity of the flow studied at the primary repeating unit-the hole-allows us to predict the global nature of the atomization process: mass transfer and spray geometry. We explain how these elementary units capture the momentum of the flow atop them and how side wall interactions can lead to saturation effects. At the grid level, this translates into surface fraction and hole aspect ratio being governing parameters of the system that can be tuned to control and optimize spray characteristics. As a result of the fragmentation, the momentum exerted on the target is reduced-a major advantage in crop protection and pathogen dispersion prevention under rain. In addition, pesticide drift in agricultural sprays can be controlled by using initially large drops that are subsequently atomized and conically sprayed by a mesh atop the crop. Beyond droplet-substrate interaction, this inexpensive spraying method enhances surface exchange phenomena such as evaporation and has major implications in many applications such as cooling towers or multieffect desalination.
\end{abstract}

DOI: 10.1103/PhysRevFluids.3.083602

\section{INTRODUCTION}

Breaking a given volume of liquid into smaller units-i.e., generating sprays-is of key importance for a wide variety of applications ranging from agriculture, coatings and paints, combustion engines, mining and waste treatment, medical, and meteorology. This bulk volume-to-droplet fragmentation can occur naturally from falling [1,2] or impacting [3-5] drops as well as bursting bubbles [6-8]. In industrial applications, it is conventionally performed by imposing a high relative velocity between the liquid to be atomized and the surrounding gas using an atomizer [9]. Although these techniques usually rely on different instabilities (Rayleigh-Plateau, Rayleigh-Taylor, and Kelvin-Helmholtz) leading to the disintegration of liquid jets and sheets [10], other methods can involve electrostatic or ultrasonic destabilization. Alternatively, drops can be atomized by impact onto a mesh or hole which leads to the transmission of part of the liquid as a spray of droplets typically 1000 times smaller than the original drop [11-16]. We observe the formation of an unexpected transmission cone that can

\footnotetext{
*david.quere@espci.fr
}

†varanasi@mit.edu 

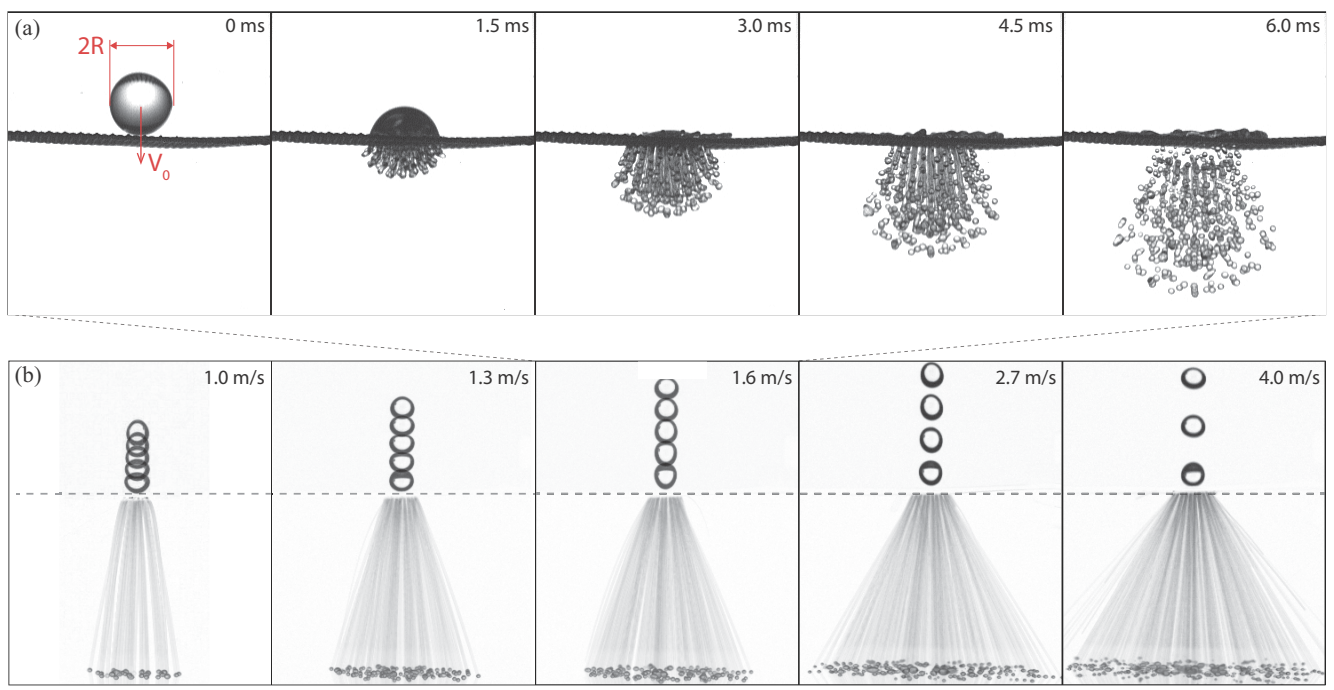

FIG. 1. (a) Time lapse of a water drop of radius $R=1.9 \mathrm{~mm}$ impacting at $V=1.6 \mathrm{~m} / \mathrm{s}$ onto a nonwetting mesh (mesh number 46 and surface fraction $\phi=0.48$ corresponding to holes of radius $b=190 \mu \mathrm{m}$ and wire diameter $d=170 \mu \mathrm{m}$ ). Time between images $1.5 \mathrm{~ms}$. (b) Chronophotography of droplet impacting on same mesh (represented by the dashed line) as in (a) for speeds $V=1.0,1.3,1.6,2.7,4 \mathrm{~m} / \mathrm{s}$, increasing from left to right. The time interval between drops before impact (region above the mesh) correspond to $2 \mathrm{~ms}$ in all cases. Third chronophotography corresponds to (a).

be tuned to control the sprayed area beneath the grid. In this system-where the liquid has a binary choice: either pass through a hole or be deflected by a plain section of the mesh-the characteristics of the spray below the mesh are dictated by its geometrical parameters as well as by the local flow profile of the crashing drop. This memory of the flow across the mesh is studied by impacting a droplet on a plate with a single hole [17]. By off-centering hole and droplet, we are able to probe the local flow at different positions and identify the key parameters governing the direction of the ejected liquid filaments (dictating the shape of the transmitted cone for a mesh) and the amount of transferred mass, two quantities relevant for industrial applications. Using a model, we are able to define a critical hole aspect ratio below which thickness can be neglected and above which we must take into account liquid rebound effects with side walls. Transmitted mass is then measured and modeled, highlighting the ability of a drop to transfer liquid even outside the area below its projected area. Extrapolating these single-hole results to the case of multiple holes allows us to tackle the more complex case of a mesh - seen as a combination of single holes-where the surface fraction of holes is found to dominate the transmission process.

\section{RESULTS AND DISCUSSIONS}

\section{A. Impact onto a mesh}

A millimetric water drop (radius $R$, density $\rho$, and surface tension $\gamma$ ) impacting at a speed $V$ of several meters per second onto a nonwetting mesh (made of woven wire of diameter $d$ and open sections of characteristic size $b$, of hundreds of microns) is illustrated in Fig. 1(a) and Movie S1 [18].

We observe how an initial volume $(\sim 30 \mu \mathrm{l})$ is divided into droplets $(\sim 0.03 \mu \mathrm{l}) 1000$ times smaller when liquid is forced through the holes of the grid. Since the time taken by the drop to flatten-which scales as $\tau \sim 2 R / V \sim 3 \mathrm{~ms}$-is larger than the Rayleigh-Plateau time-which scales as $\sqrt{\rho b^{3} / \gamma} \sim 0.3 \mathrm{~ms}$-liquid filaments destabilize into tiny droplets forming a conical spray where edges are defined by the angle of ejection $\alpha$. Figure 1(b) shows a chronophotography of how 
(a)

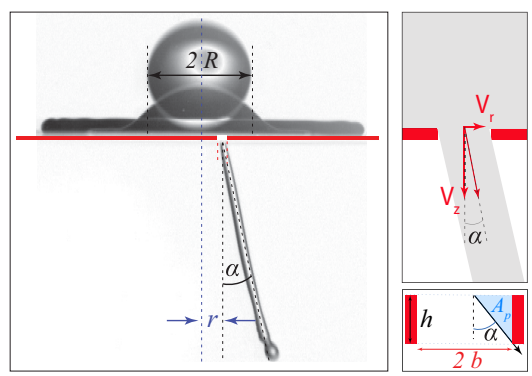

(c)

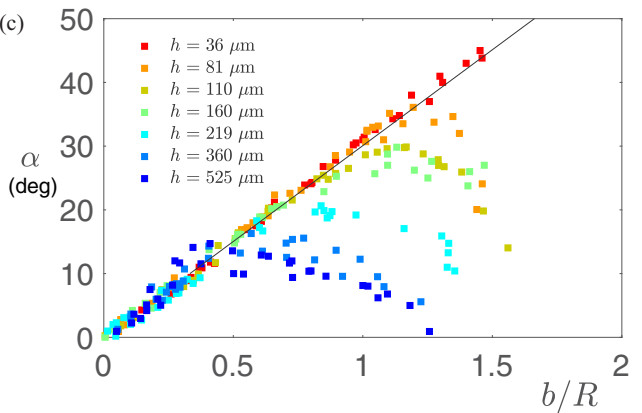

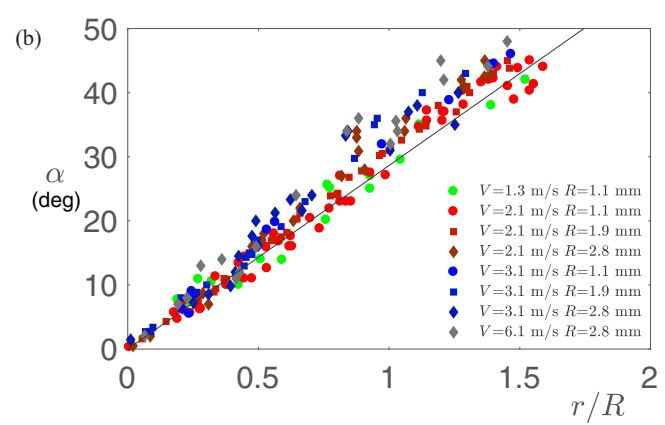

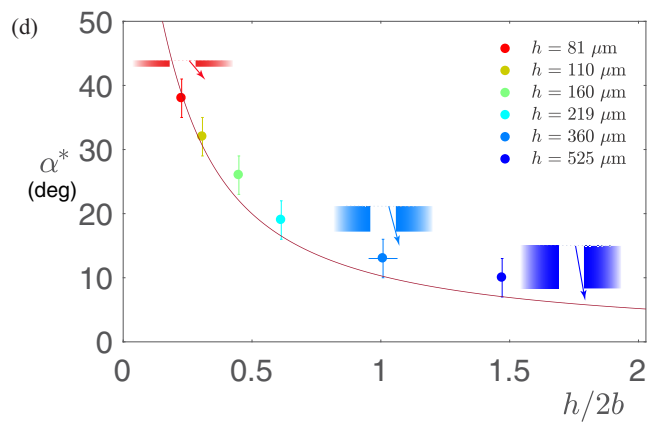

FIG. 2. (a) Chronophotography of a drop ( $R=1.1 \mathrm{~mm}, V=2.1 \mathrm{~m} / \mathrm{s})$ impacting a plate of thickness $h=$ $36 \pm 5 \mu \mathrm{m}$ pierced with a hole of radius $b=180 \pm 10 \mu \mathrm{m}$ at an off-centered position $r=0.44 R$. We can observe a liquid filament ejected at an angle $\alpha$. Right top panels show a zoomed sketch of the hole area. Right bottom panel shows a cross-sectional sketch of the hole. (b, c) Angle of ejection $\alpha$ as a function of the normalized off-centering position of the hole $r / R$. Black line shows Eq. (1). Hole radius $b=180 \pm 10 \mu \mathrm{m}$. (b) Speed $V$ and drop radius $R$ are varied, while plate thickness $h=36 \pm 5 \mu \mathrm{m}$ is kept constant. (c) Speed $V=2.1 \mathrm{~m} / \mathrm{s}$ and drop radius $R=1.9 \mathrm{~mm}$ are kept constant, while plate thickness $h$ is varied. (d) Critical angle $\alpha^{*}$ as a function of the hole aspect ratio $h / 2 b$. Solid line shows Eq. (2) with $A_{p} / A^{*}=10 \%$. Sketches show the real hole aspect ratio for fixed radius $b=180 \pm 10 \mu \mathrm{m}$ and thickness $h=81$ (red), 160 (light blue), and 525 (blue) $\mu \mathrm{m}$. The arrow in the sketch shows $\alpha^{*}$ and divides the cross section of the hole so that the perturbed area corresponds to $10 \%$.

this envelope depends on the impacting speed and results in the spreading of the grated droplets over an area bigger than the original projected area of the drop. While the meshes used in this study are nonwetting, the behavior of drops impacting on plain meshes is similar [18]. In order to understand the elements governing the relative amount of transferred mass $m / m_{0}\left(m_{0}\right.$ and $m$ being the initial and transmitted mass, respectively) and the angle of ejection $\alpha$, crucial in many applications, we first study the case of a drop impacting on a single hole.

\section{B. Impact on a single hole: Ejection angle $\alpha$}

When a drop impacts a nonwetting plate of thickness $h$ pierced with a hole of radius $b$ off-centered by a distance $r$ from the axis of symmetry of the drop [see Fig. 2(a) and Movie S2 [18]] an equivalent behavior is observed. If impacting speed $V$ is greater than the critical speed $V^{*}=2 \sqrt{\gamma / \rho b}$ - set by the balance between inertial $\rho V^{2}$ and capillary $\gamma / b$ pressure [17]_liquid can be pushed through the hole. Indeed, the capillary number, $\mathrm{Ca}=\mu V / \sigma$, is below 0.1 for all the velocities considered, indicating that viscosity can be neglected against capillary forces.

Assuming an hyperbolic flow profile for the flow in the crashing drop [19], the integral form of the incompressibility expression gives us a relation between vertical $V_{z}$ and horizontal $V_{r}$ components 
of the speed [see Fig. 2(a)]: $V_{r}=-\frac{r}{2} \frac{\partial V_{z}}{\partial z}$. If we consider that the angle of ejection is set by the ratio of these speeds $\alpha=\arctan V_{r} / V_{z}$, we get the following scaling law:

$$
\alpha \sim \frac{r}{2 R} \text {, }
$$

where we have used $R$ as the vertical length scale and assumed the small angles approximation $\tan x \sim x$. To experimentally confirm Eq. (1), we compare in Fig. 2(b) the angle of ejection $\alpha$ for different speeds $V \in 1.3,2.1,3.1,6.1 \mathrm{~m} / \mathrm{s}$ and drop radius $R \in 1.1,1.9,2.8 \mathrm{~mm}$. As predicted, we observe a linear dependence of $\alpha$ versus the off-centering distance normalized by the natural length scale of the droplet $r / R$ and an invariance versus the velocity $V$. All experiments in Fig. 2(b) are conducted with a hole of high aspect ratio where the horizontal dimension $(2 b=360 \pm 20 \mu \mathrm{m})$ is one order of magnitude greater than the vertical one $(h=36 \pm 5 \mu \mathrm{m})$ so that we can neglect any thickness effect and assume an infinitely thin plate. To investigate the role of thickness we show in Fig. 2(c) the angle of ejection as a function of $r / R$ for increasing thickness $h$, keeping $V=2.1 \mathrm{~m} / \mathrm{s}$ and $R=1.9 \mathrm{~mm}$ fixed. In this case, we observe the appearance of a critical saturation angle $\alpha^{*}$ defined as the angle for which data start to deviate from the infinitely thin plate model. To understand this saturation, we define a cross-sectional area $A_{p}=\frac{1}{2} h^{2} \tan \alpha$ [shaded blue in bottom right panel in Fig. 2(a)] where the trajectories of the fluid particles entering the hole would intersect with the side walls and be perturbed. The ratio of the perturbed to total area $A=2 b h$ is hence $A_{p} / A=\tan \alpha h / 4 b$. If we have a high aspect ratio hole $(h \ll b), A_{p} / A \rightarrow 0$, and we are in the infinitely thin plate regime. In the opposite case, $A_{p} / A \rightarrow 1$, and all the fluid can be assumed to be perturbed by the wall thickness. With this simple definition, we can now define a critical value of perturbed fluid percentage $A_{p} / A^{*}$ over which we start to deviate from the infinitely thin plate regime:

$$
\alpha^{*}=\arctan \frac{4 b}{h} \frac{A_{p}{ }^{*}}{A} .
$$

To compare this model with the data we show in Fig. 2(d) $\alpha^{*}$ as a function of the hole's aspect ratio $h / 2 b$. Equation (2) (solid red line) shows best experimental agreement for $\frac{A_{p}{ }^{*}}{A}=0.1$ (fixed value for all data). As a result, the infinitely thin plate approximation is valid only for perturbed areas smaller than $10 \%$. In addition, Eq. (2) allows us to predict the saturation value $\alpha^{*} \approx 63^{\circ}$ for the mesh used in Fig. 2(a) and validate the use of the infinitely thin plate model in Eq. (1). The overall trend ejection angle after deviating from the linear behavior can be explained by assuming an elastic rebound against the lateral walls: the perturbed liquid amount will first saturate the ejection angle, and then it will overcome it, leading to a decrease as shown in Fig. 2(c).

We also observe in Fig. 2 that although transmission is possible outside the projected area of the droplet (see data for $r>R$ ) there is an off-centering distance upon which liquid transfer ceases (no data for $r>1.5 R$ ). In order to investigate what sets these transitions, we focus on the measure of the transmitted mass developed in the next section.

\section{Impact on a single hole: Mass transfer}

Transmitted mass through a single hole is measured with a high sensitivity microbalance $(1 \mu \mathrm{g}$ resolution). In the case of a centered impact $r=0$, we vary drop and holes sizes as well as impacting speed. Since it takes a time $\tau \sim 2 R / V$ for the drop to crash, the total volume transferred is $Q \tau$, where $Q=\left(V-V^{*}\right) \pi b^{2}$ is the volumetric flow through the hole. Indeed, if we interpret $V^{*}$ as a Taylor-Culick retraction speed [20,21] of the liquid finger formed at the hole exit, if $V<V^{*}$ no mass is transferred, and if $V>V^{*}$ liquid is transmitted at speed $V-V^{*}$. As a result, we can express the relative amount of transmitted mass as

$$
\frac{m}{m_{0}}=\frac{3}{2}\left(\frac{b}{R}\right)^{2}\left(1-\frac{V^{*}}{V}\right) .
$$

When comparing this model to experiments [Fig. 3(a)] we observe a collapse of the data over several orders of magnitude, corresponding to masses ranging from tens of micrograms for the 


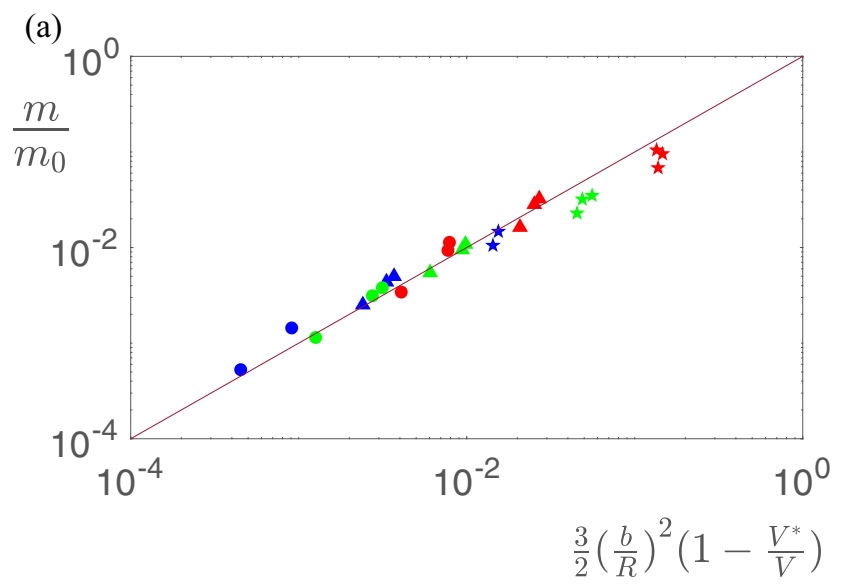

(b)

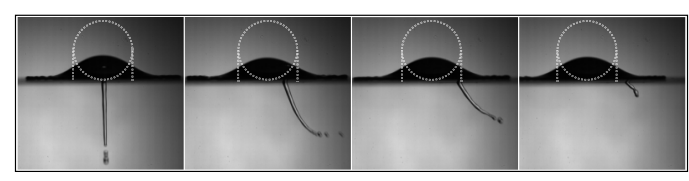

(c)

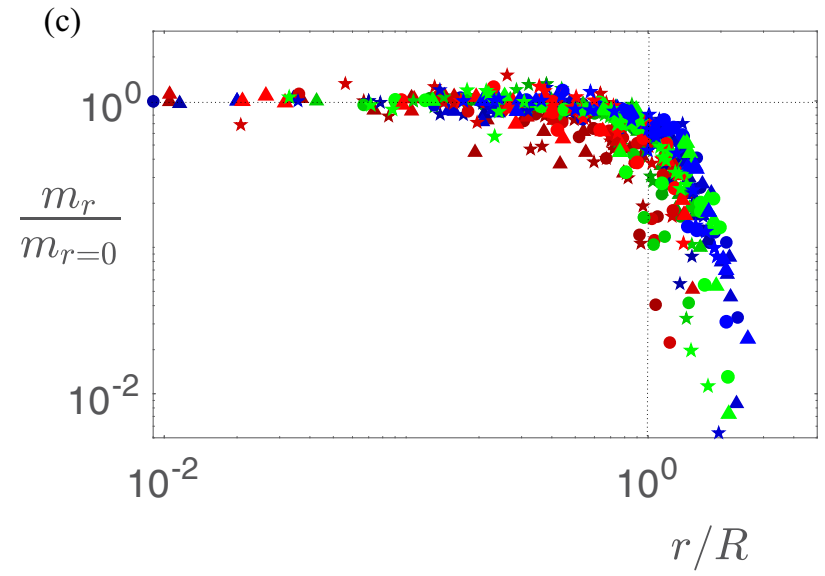

FIG. 3. (a) Transmitted mass percentage as a function of the model prediction [Eq. (3)] for centered holes $r=0 \pm 0.2 \mathrm{~mm}$. Marker type describes hole size $b: 110 \pm 5 \mu \mathrm{m}$ in circle, $180 \pm 10 \mu \mathrm{m}$ in triangle, $320 \pm$ $10 \mu \mathrm{m}$ in star. Color describes drop radius $R: 1.1 \mathrm{~mm}$ in red, $1.9 \mathrm{~mm}$ in green, $2.8 \mathrm{~mm}$ in blue. For a given $R$ and $b$, three speeds were used: $1.4,2.5,3.4 \mathrm{~m} / \mathrm{s}$. Red line shows equation $y=x$. (b) Side view of drop $(R=1.9 \mathrm{~mm}$, $V=2.2 \mathrm{~m} / \mathrm{s})$ impacting on a hole $(b=180 \pm 10 \mu \mathrm{m})$ with increasing off-centering parameter $r / R: 0,0.65,0.95$, 1.45 from left to right. Image is taken $1.5 \mathrm{~ms}$ after initial touchdown in all cases. White dashed line shows initial drop position and volume. (c) Transmitted mass $m_{r}$ as a function of the off-centering parameter $r / R$ normalized by the center case $m_{r=0} . m_{r}$ ranges over two orders of magnitude: from tens to thousands of $\mu \mathrm{g}$. Same marker and color definition as in (a). Dashed lines: Vertical shows $r / R=1$ after which rapid decrease of liquid transfer is observed. Horizontal shows maximum transmission happening when $r=0$, the case seen in Fig. 3(a).

smallest drop, speed, and hole size to thousands of micrograms in the opposite case. Although impacting speed has a strong effect in the vicinity of $V^{*}$, once $V \gg V^{*}$, relative mass transmission saturates to its maximum value set by the ratio of the hole to drop area $(b / R)^{2}$. 
We can observe in Fig. 3(b) the effect of the off-centering parameter $r$ while keeping all other parameters constant. If $r / R<1$ (first three pictures), transmitted mass is similar to the centered case. If $r / R>1$ (last picture), mass is still transferred but rapidly decreases as off-centering increases. This effect is quantitatively shown in Fig. 3(c) where transmitted mass $m_{r}$ normalized by the centered case $m_{r=0}$, is plotted as a function of $r / R$. We observe that although we vary drop speed $V$, drop size $R$, and hole size $b$ (so that $m_{r}$ ranges over two orders of magnitude: from tens to thousands of micrograms), rescaled transmission follows a similar behavior, owing to the self-similarity of the flow [19] inside a crashing droplet. Furthermore, data can be divided into two domains. In the first one, the hole is under the projected area of the drop and transmission plateaus to a value similar to the centered scenario. In this case we can assume that the local speed at the hole is similar to the impacting speed $V$. In a second domain, the hole is outside the projected area, and, although transmission is still possible close to $r \geqslant R$, it decreases rapidly with $r$. In this case, local speed at the hole is smaller due to the deceleration of the drop during spreading. In our study, we observe a typical maximum value of $r_{\max } / R \approx 1.5 \pm 0.2$, consistent with the ejection angle study (no measurable values for $r$ greater than $r / R=1.5$ ). In order to get a more detailed model, an exact resolution of the local speed as a function of $r$ would be needed, which is still an open problem [19,22].

\section{Impact on a mesh: The case of multiple holes}

With the understanding of the governing mechanisms at primary unit level-the hole-we tackle the more complex case of the mesh. We study a range of meshes (geometrical parameters in Ref. [18]) for which the drop size is typically 10-100 times greater than the holes size so that the findings of a single hole can be extrapolated to it. Indeed, if the drop sees too few holes, i.e., the size of the open or closed areas is similar or greater than the size of the drop $(d+2 b) / 2 R \sim 1, b$ being the distance between wires making the woven mesh and $d$ their diameter [see Fig. 4(a)], we are closer to the case of a drop impacting a fiber [23,24] or a plate [19]. If it sees too many holes, $(d+2 b) / 2 R \ll 1$, additional effects come into play such as hydrodynamic focusing, or water hammer pressure [11,12]. In terms of number of holes per inch (or mesh number), a parameter conventionally used to describe meshes together with the open area fraction $\phi=1 /(1+d / 2 b)^{2}$, this translates into a mesh number ranging from 20 to 200 , values corresponding to what is most generally used in industrial and commercial applications.

Regarding cone angle $\alpha$, we observe in Fig. 4(b) (top left) that its variation appears independent of the mesh number, all curves saturating at a similar value of $19^{\circ}$. Since meshes are woven with circular wires $(h=d)$, hole aspect ratio can be expressed as $h / 2 b=1 / \sqrt{\phi}-1$. Using Eq. (2) we get

$$
\alpha^{*} \sim \arctan \frac{2}{1 / \sqrt{\phi}-1} \frac{A_{p}{ }^{*}}{A} .
$$

As a consequence, for a constant $\phi=0.31$ we expect a constant critical saturation angle $\alpha^{*}=14^{\circ}$ Although this value is close to the experimental one observed in Fig. 4(b), it is lower due to the curved geometry of the woven grid hole's walls, a less constrained system than the modeled vertical walls used to establish Eq. (2) (see in Ref. [18] a complete model taking into account wall curvature with $\alpha^{*}{ }_{c}$ ). Symmetrically, if we increase $\phi$ while keeping mesh number constant $[\#=40$, the case shown in top right of Fig. 4(b)], saturation is pushed to higher values, and we can observe the appearance of a dependency of $\alpha$ towards impacting speed $V$ close to $V^{*}$. Since angle of ejection can be related back to an off-centering position, this means that for the case of fairly open meshes [see red curve $\phi=0.55$ in Fig. 4(b) right, for which we expect saturation around $\alpha^{*}{ }_{c}=42^{\circ}$ ] the area over which mass is transmitted depends on impacting conditions.

In order to further investigate this dependency, we study the transfer mechanism by measuring the transmitted mass [bottom of Fig. 4(b)] and construct a simple model. If we define $R_{t}$ as the radius of area over which liquid transfer occurs [shaded blue area in Fig. 4(a)] we have $N_{t}=\phi R_{t}^{2} / b^{2}$ 
(a)

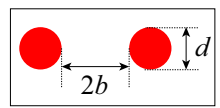

(b)

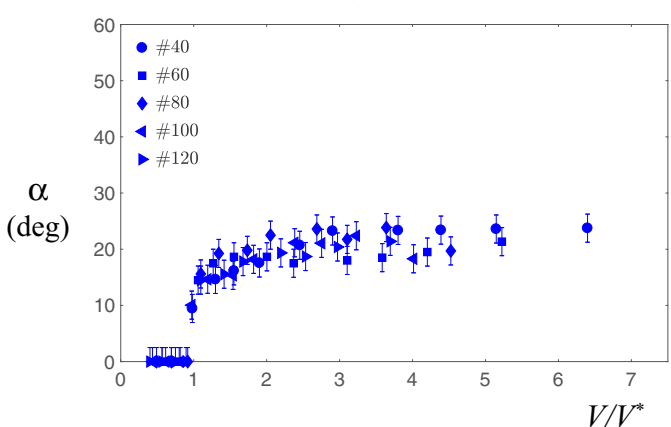

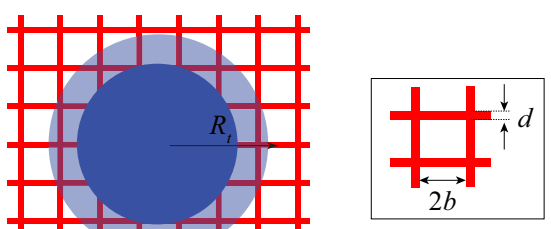

\# 40

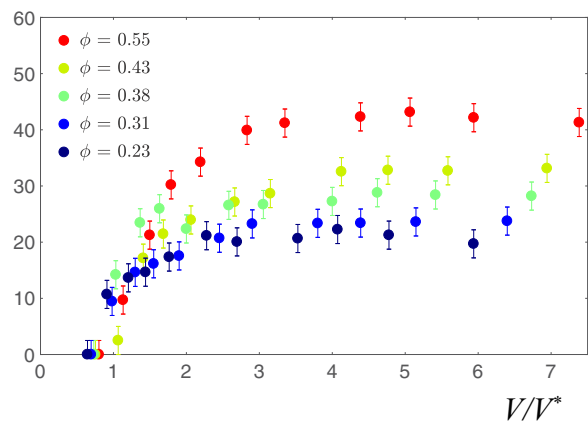

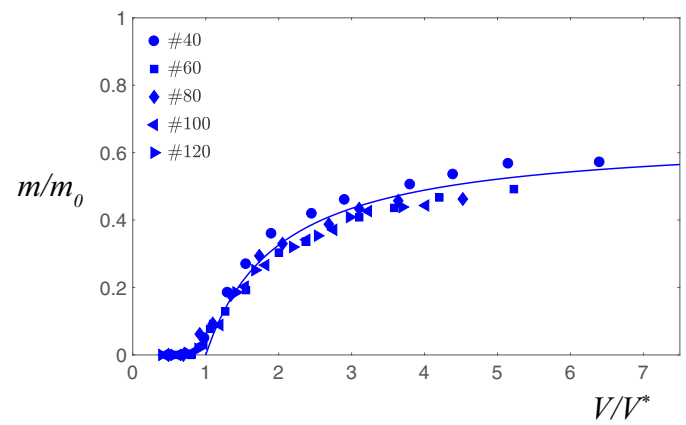

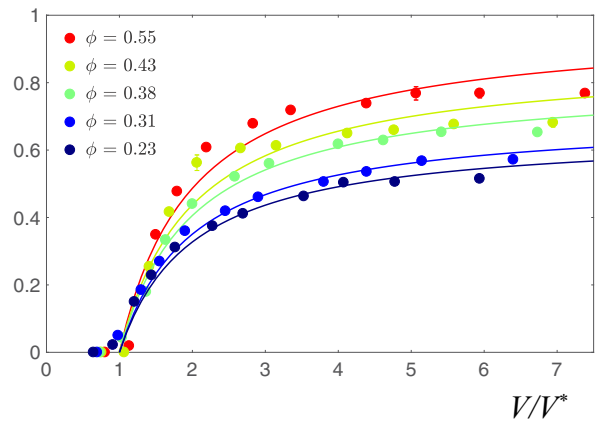

FIG. 4. (a) Side (left half) and top (right half) sketch of drop-mesh system. Insets show close-up view detail of geometrical parameters of the grid. (b) Angle of ejection (top row) and transmitted mass percentage (bottom row) for a drop $(R=2.1 \mathrm{~mm})$ impacting a mesh of constant surface fraction of holes $(\phi=0.31$ left column $)$ or constant mesh number (\# = 40, right column) as a function of normalized impacting speed. In the case of mass transmission (bottom), solid lines show best fit following Eq. (5) (the resulting prefactor being 1.1 \pm 0.2 ). “\#” in left panels of (b) is mesh number.

transmitting holes. Using Eq. (3) we obtain for the mesh

$$
\frac{m}{m_{0}} \sim\left(\frac{R_{t}}{R}\right)^{2} \phi\left(1-\frac{V^{*}}{V}\right) .
$$

As observed in Fig. 4(b) (bottom left), Eq. (5) predicts that transmission is independent of mesh number. In addition, similar to the single-hole experiments, mass transfer increases with impacting speed until it reaches a saturation value proportional to surface fraction $\phi$ and normalized transfer area $\left(\frac{R_{t}}{R}\right)^{2}$. Using the observations for the single hole, we can approximate $R_{t} / R$ by $r_{\max } / R \approx 1.5$. As a result, we see a good agreement with data in Fig. 4(b) where best fit following Eq. (5) is shown in solid line (the associated prefactor being $1.1 \pm 0.2$ ). Again, although outside the scope of this work, a more detailed expression for the normalized transfer area would require an exact resolution 
(a)
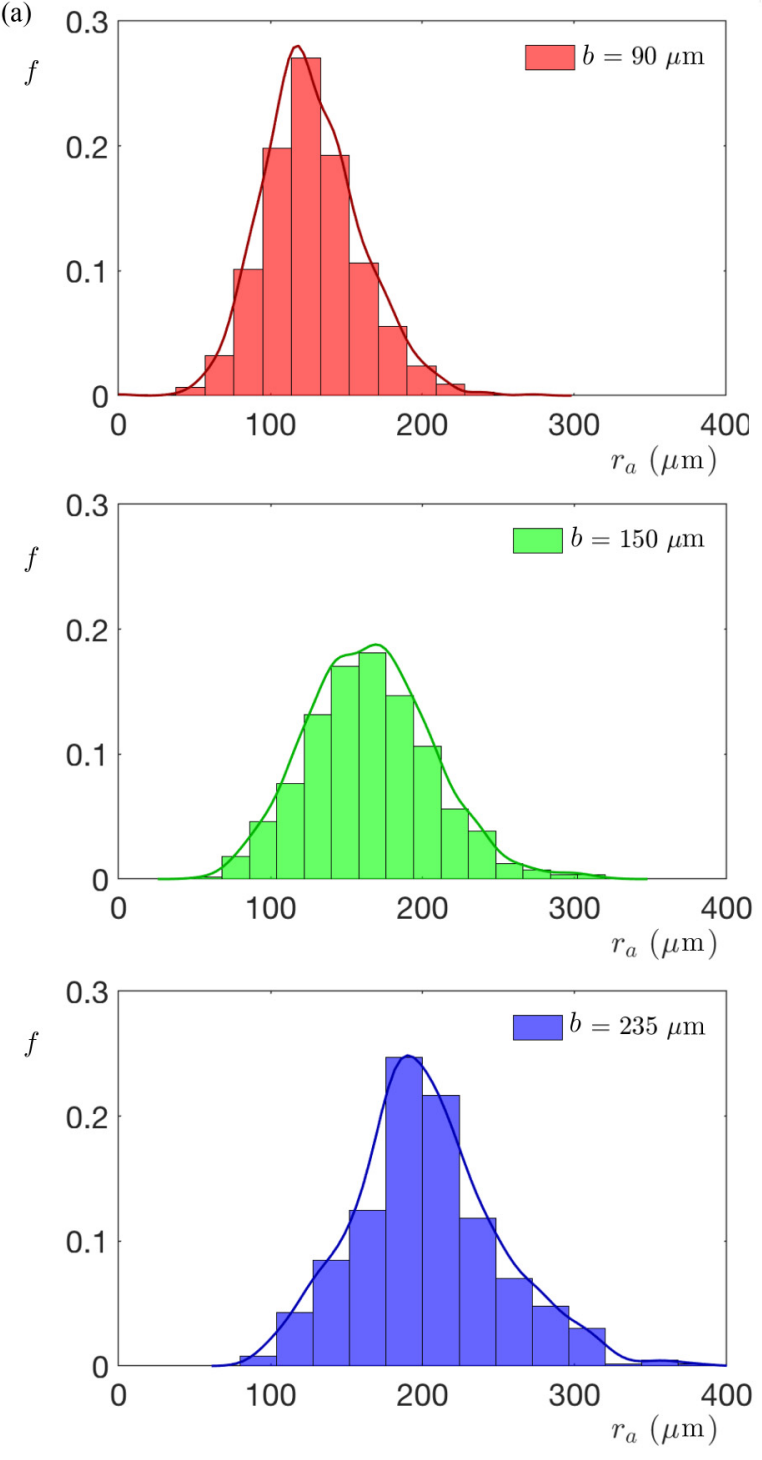
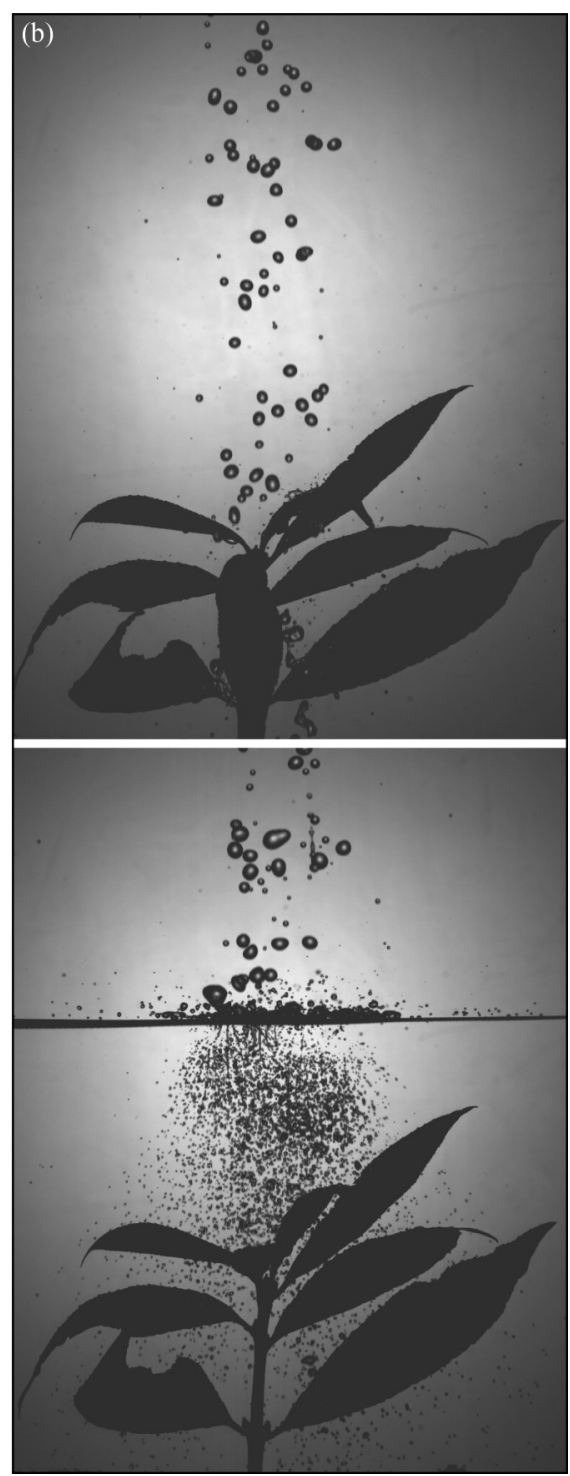

FIG. 5. (a) Atomized droplet radius, $r_{a}$, distribution for drop with velocity $V / V^{*}=3$ impacting a mesh with opening size $b=90,150$, and $235 \mu \mathrm{m}$ respectively. (b) Rain of millimetric drops impacting at speed $V=3 \mathrm{~m} / \mathrm{s}$ directly onto the leaves of a plant (top) or through a mesh [bottom, $\phi=0.55$, number $=40$, corresponding to red dots in Fig. 4(b)]. We can observe how in the second case a spray of much finer droplets is generated in situ - typically reducing the momentum of the droplets by a factor of 1000 - at the same time that the incoming rain is spread over a larger area.

of the local speed profile atop each hole (taking into account purging effects or dependency of $R_{t}$ towards $V$ ).

To evaluate the efficiency of the atomization process, we show in Fig. 5(a) the distribution of the atomized droplets radii, $r_{a}$, generated by the impact of a drop on a mesh with opening size $b=90$, 150 , and $235 \mu \mathrm{m}$, respectively. To enable comparison of the results between the different meshes, the Weber number relative to the mesh holes was kept constant such that $V / V^{*}=3$ in all cases. This velocity corresponds to reaching the plateau in mass transmission shown in Fig. 4(b) and is 
therefore of interest for applications. The atomized droplets size distributions exhibit a peak at 118 , 165 , and $190 \mu \mathrm{m}$, respectively, with a standard deviation of $10 \%$. These values are close to the size of the respective opening, indicating that the droplets are generated from the Rayleigh-Plateau destabilization of the jets going through the holes. Note that the peak droplet radius is larger than the hole radius for the smaller holes while it is smaller than the hole radius for the largest opening size. This effect, while outside the scope of this study, is due to the dynamics of jet destabilization and has been extensively studied elsewhere $[25,26]$.

We can observe in Fig. 5(b) and Movie S3 [18] raindrops falling onto the leaves of a plant with and without a mesh that atomizes them in situ. By breaking drops into thousands of smaller droplets, we limit by an identical factor the momentum exerted on the target. In particular, Fig. 5(a) shows that no droplets larger than $350 \mu \mathrm{m}$ in radius are created, thereby greatly reducing the deflection of the leaf (see Movie S3 [18]), a considerable advantage for agriculture purposes where crop damage and pathogen dispersion caused by heavy rains is a major problem $[27,28]$.

Beyond preventing disease spreading, this approach paves the way for new solutions in agricultural spraying. Indeed, it allows one to tackle the problem of pesticide drift [29] by initially spraying larger drops that are atomized at a final stage, only close to the crop. The resulting spray leads to a broader coverage area due to the spreading effect of the mesh [see Fig. 5(b) where incoming rain laterally constrained to an initial column streak is enlarged after atomization, wetting areas that would have otherwise remained dry] and has a higher deposition efficiency because fine droplets with small velocity have a higher probability to stick to the leaves. By tuning the impact and mesh parameters, droplet rebound on the mesh [16] can be avoided and the transmitted mass can be maximized. In addition, drop impact on meshes can be used to increase surface-to-volume ratio without additional energy input, which leads to an enhancement of surface exchange processes such as evaporation. As a consequence, this work enables new approaches in evaporative processes as varied as cooling towers or multi-effect desalination. Finally, the mesh atomization approach can be generalized to any spraying process where clogging and aging of nozzles is a problem. By considering a two-step process - generating first a spray of big drops (where clogging is less problematic) and then refining it with a mesh — we can potentially reduce maintenance and repair costs of finer nozzles and enhance overall process efficiency.

\section{ACKNOWLEDGMENTS}

We thank MIT-France program for financial support, R. Cohen and S. Srinivasan for useful discussion and mesh samples, and R. Morris for valuable comments. The authors declare that they have no competing interests.

[1] R. H. Magarvey and B. W. Taylor, Shattering of large drops, Nature (London) 177, 745 (1956).

[2] E. Villermaux and B. Bossa, Single-drop fragmentation determines size distribution of raindrops, Nat. Phys. 5, 697 (2009).

[3] J. C. Bird, R. Dhiman, H.-M. Kwon, and K. K. Varanasi, Reducing the contact time of a bouncing drop, Nature (London) 503, 385 (2013).

[4] A. Gauthier, S. Symon, C. Clanet, and D. Quéré, Water impacting on superhydrophobic macrotextures, Nat. Commun. 6, 8001 (2015).

[5] C. Josserand and S. T. Thoroddsen, Drop impact on a solid surface, Annu. Rev. Fluid Mech. 48, 365 (2016).

[6] X. Wang et al., The role of jet and film drops in controlling the mixing state of submicron sea spray aerosol particles, Proc. Natl. Acad. Sci. U.S.A. 114, 6978 (2017).

[7] H. Lhuissier and E. Villermaux, Bursting bubble aerosols, J. Fluid Mech. 696, 5 (2012).

[8] J. C. Bird, R. de Ruiter, L. Courbin, and H. A. Stone, Daughter bubble cascades produced by folding of ruptured thin films, Nature (London) 465, 759 (2010).

[9] H. Lefebvre and V. G. McDonell, Atomization and Sprays (CRC Press, Boca Raton, FL, 2017). 
[10] N. Rimbert and G. Castanet, Crossover between Rayleigh-Taylor instability and turbulent cascading atomization mechanism in the bag-breakup regime, Phys. Rev. E 84, 016318 (2011).

[11] S. Ryu, P. Sen, Y. Nam, and C. Lee, Water Penetration through a Superhydrophobic Mesh during a Drop Impact, Phys. Rev. Lett. 118, 014501 (2017).

[12] P. Brunet, F. Lapierre, F. Zoueshtiagh, V. Thomy, and A. Merlen, To grate a liquid into tiny droplets by its impact on a hydrophobic microgrid, Appl. Phys. Lett. 95, 254102 (2009).

[13] C. F. Hsu and N. Ashgriz, Impaction of a droplet on an orifice plate, Phys. Fluids 16, 400 (2004).

[14] J. Xu, J. Xie, X. He, Y. Cheng, and Q. Liu, Water drop impacts on a single-layer of mesh screen membrane: Effect of water hammer pressure and advancing contact angles, Exp. Therm. Fluid Sci. 82, 83 (2017).

[15] R. P. Sahu, S. Sett, A. L. Yarin, and B. Pourdeyhimi, Impact of aqueous suspension drops onto non-wettable porous membranes: Hydrodynamic focusing and penetration of nanoparticles, Colloids Surfaces A 467, 31 (2015).

[16] A. Kumar, A. Tripathy, Y. Nam, C. Lee, and P. Sen, Effect of geometrical parameters on rebound of impacting droplets on leaky superhydrophobic meshes, Soft Matter 14, 1571 (2018).

[17] É. Lorenceau and D. Quéré, Drops impacting a sieve, J. Colloid Interface Sci. 263, 244 (2003).

[18] See Supplemental Material at http://link.aps.org/supplemental/10.1103/PhysRevFluids.3.083602 for methods, mesh information, derivation of the saturation ejection angle in the case of woven meshes, and videos.

[19] G. Lagubeau et al., Spreading dynamics of drop impacts, J. Fluid Mech. 713, 50 (2012).

[20] F. E. C. Culick, Comments on a ruptured soap film, J. Appl. Phys. 31, 1128 (1960).

[21] C. Clanet and J. C. Lasheras, Transition from dripping to jetting, J. Fluid Mech. 383, 307 (1999).

[22] S. S. Kumar, A. Karn, R. E. A. Arndt, and J. Hong, Internal flow measurements of drop impacting a solid surface, Exp. Fluids 58, 12 (2017).

[23] E. Dressaire, A. Sauret, F. Boulogne, and H. A. Stone, Drop impact on a flexible fiber, Soft Matter 12, 200 (2016).

[24] S.-G. Kim and W. Kim, Drop impact on a fiber, Phys. Fluids 28, 042001 (2016).

[25] J. Eggers and E. Villermaux, Physics of liquid jets, Rep. Prog. Phys. 71, 36601 (2008).

[26] W. van Hoeve, S. Gekle, J. H. Snoeijer, M. Versluis, M. P. Brenner, and D. Lohse, Breakup of diminutive Rayleigh jets, Phys. Fluids 22, 122003 (2010).

[27] T. Gilet and L. Bourouiba, Fluid fragmentation shapes rain-induced foliar disease transmission, J. R. Soc. Interf. 12, 20141092 (2015).

[28] T. Gilet and L. Bourouiba, Rain-induced ejection of pathogens from leaves: Revisiting the hypothesis of splash-on-film using high-speed visualization, Integr. Comp. Biol. 54, 974 (2014).

[29] E. Hilz and A. W. P. Vermeer, Spray drift review: The extent to which a formulation can contribute to spray drift reduction, Crop Prot. 44, 75 (2013). 